\title{
Numerical solution of fuzzy initial value problem (FIVP) using optimization
}

\author{
Ali Asghar Behroozpoor*, Ali Vahidian Kamyad, Mohammad Mehdi Mazarei \\ Department of Applied Mathematics, School of Mathematical Sciences, Ferdowsi University of Mashhad, International Campus, \\ Mashhad, Iran
}

\section{A R T I C LE I N F O}

\section{Article history:}

Received 2 June 2016

Received in revised form

13 August 2016

Accepted 16 August 2016

\section{Keywords:}

Fuzzy

Initial value problem

Optimization

Linear programming

Numerical solution

\begin{abstract}
A B S T R A C T
In this paper, we introduce a new approach to obtain a novel numerical solution of fuzzy initial value problem (FIVP). This technique is based on optimization problem. In fact, the optimal solution of optimization problem is approximated solution of fuzzy initial value problem. Theoretical consideration is discussed and some examples are presented to show the ability of the method for fuzzy initial value problem.
\end{abstract}

(C) 2016 The Authors. Published by IASE. This is an open access article under the CC BY-NC-ND license (http://creativecommons.org/licenses/by-nc-nd/4.0/).

\section{Introduction}

In modeling of real physical phenomena, differential equations play an important role in many areas of science and engineering. In many cases, information about the physical phenomena involved is always pervaded with uncertainty. Fuzzy differential equations (FDEs) are a natural way to model dynamical systems under possibilistic uncertainty. Also, in modeling real-word phenomena, fuzzy initial value problems (FIVP) appear naturally.

Fuzzy linear systems have recently been studied by a good number of researchers, but only a few of them are mentioned here. Friedman et al. (1998), Allahviranloo (2004, 2005a, 2005b) and Annelies Vroman et al. (2008).

The concept of fuzzy derivative was first introduced by Chang and Zadeh in (1965) it was followed up by Dubios and prade (1982), who defined and used the extension principle. The fuzzy differential equation and the fuzzy initial value problem were regularly treated by Kaleva in (1987, 1990) and by Seikkala in (1987).

There are several approaches to the study of fuzzy differential equations. The approach based on H-derivative (Puri and Ralescu, 1983) has the disadvantage that any solution of a FDE has increasing length of its support (Diamond, 2000).

\footnotetext{
* Corresponding Author.

Email Address: abehroozpoor@yahoo.com (A. Behroozpoor) http://dx.doi.org/10.21833/ijaas.2016.08.007

2313-626X/@ 2016 The Authors. Published by IASE.

This is an open access article under the CC BY-NC-ND license (http://creativecommons.org/licenses/by-nc-nd/4.0/)
}

This shortcoming was resolved by interpreting a FDE as a family of differential inclusions (Hullermeier, 1999). The main shortcoming of using differential inclusions is that we do not have a derivative of a fuzzy -number-valued function, and so, the numerical solutions of a FDE are difficult to be obtained. Strongly generalized differentiability of fuzzy -number-valued functions is introduced and studied in (Bede and Gal, 2005). The numerical method for solving fuzzy differential equations is introduced in (Ma et al., 1999; Abbasbandy and Allahviranloo, 2002; Abbasbandy et al., 2004; Allahviranloo et al., 2007; Allahviranloo et al., 2006) by standard Euler method.

In this paper, we have used a different method to obtain the numerical solution of fuzzy initial valued problem (FIVP). In this method, we reduce (FIVP) to an optimization problem. In fact, the optimal solution of this optimization problem is the numerical solution of FIVP. Also, this method is simple and it does not need any differentiability of the fuzzy functions.

\section{Preliminaries}

\subsection{Fuzzy sets}

According to zadeh (1965), a fuzzy set is a generalization of a classical set that allows membership

Definition 2.1.1: Let $U$ be a universal set. A fuzzy set $\tilde{A}$ in $U$ is defined by a membership function $\mu_{\tilde{A}}$ that maps every element in $U$ to the unit interval $[0,1]$. A fuzzy set $\tilde{A}$ in $U$ may also be presented as a set of ordered pairs of a generic element $x$ and its 
membership value, as shown in the following equation:

$$
\tilde{A}=\left\{\left(x, \mu_{\tilde{A}}(x)\right): x \in U\right\}
$$

Definition 2.1.2: The support of a fuzzy set $\tilde{A}, S(\tilde{A})$ is the crisp set of all $x \in U$ such that $\mu_{\tilde{A}}(x)>0$.

Definition 2.1.3: Let $\tilde{A}$ be a fuzzy set defined in $U$. The core of $\tilde{A}$ is the crisp set of all elements in $U$ such that the membership value of $\tilde{A}$ is 1 , that is:

$$
\operatorname{core}(\tilde{A})=\left\{x \in U \mid \mu_{\tilde{A}}(x)=1\right\}
$$

Definition 2.1.4: The (crisp) set of elements that belong to a fuzzy set $\tilde{A}$ at least to the degree $\alpha$ is called the $\alpha$-level set:

$$
A_{\alpha}=\left\{x \in U \mid \mu_{\tilde{A}}(x) \geq \alpha\right\}
$$

Definition 2.1.5: A fuzzy set $\tilde{A}$ is convex if

$$
\begin{aligned}
& \mu_{\tilde{A}}\left(\lambda x_{1}+(1-\lambda) x_{2}\right) \geq \\
& \min \left\{\mu_{\tilde{A}}\left(x_{1}\right), \mu_{\tilde{A}}\left(x_{2}\right)\right\} ; x_{1}, x_{2} \in U, \lambda \in[0,1]
\end{aligned}
$$

Alternatively, a fuzzy set is convex if all $\alpha$-cut sets are convex.

Definition 2.1.6: A fuzzy number $\tilde{A}$ is a convex normalized set $\tilde{A}=(\underline{A}, \bar{A})$ of the real line $\mathbb{R}$ such that:

1) It exist exactly one $x_{0} \in \mathbb{R}$ with $\mu_{\tilde{A}}(x)=1$.

2) $\mu_{\tilde{A}}(x)$ is piecewise continuous.

Nowadays, definition 2.6 is very often modified.

Definition 2.1.7: A triangular fuzzy number has the following form:

$$
\mu_{\tilde{A}}(x)=\left\{\begin{array}{lc}
0 & x<a \\
\frac{x-a}{m-a} & a \leq x \leq m \\
\frac{b-x}{b-m} & m \leq x \leq b \\
0 & b<x
\end{array}\right.
$$

A triangular fuzzy number is denoted by $\tilde{A}=(m, a, b)$ where, $c \neq a, c \neq b$. For a triangular fuzzy number, we have:

$$
\underline{A}(\alpha)=a+(c-a) \alpha
$$

and

$$
\bar{A}(\alpha)=b+(c-b) \alpha
$$

$a$ and $b$ are called left and right spreads of the fuzzy number $\tilde{A}$. Fig. 1 shows a triangular fuzzy number.

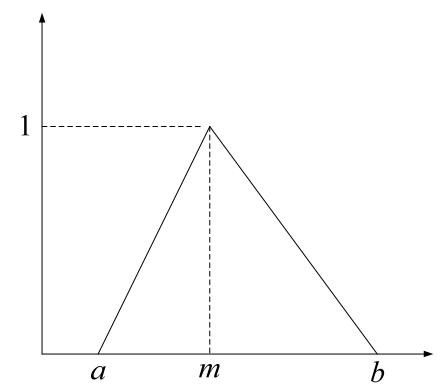

Fig. 1: The figure of triangular fuzzy number $\tilde{A}$

Definition 2.1.8: A fuzzy number $\tilde{A}$ is called positive (negative) if its membership function is such that $\mu_{\tilde{A}}(x)=0, \forall x<0(\forall x>0)$.

\subsection{The extension principle}

One of the most basic concepts of fuzzy set theory that can be used to generalize crisp mathematical concepts to fuzzy sets is the extension principle.

Definition 2.2.1: Let $X=X_{1} \times X_{2} \times \ldots \times X_{r}$ and $\tilde{A}_{1}, \tilde{A}_{2}, \ldots, \tilde{A}_{r}$ be $r$ fuzzy sets in $X_{1}, X_{2}, \ldots, X_{r}$, respectively. $f$ is a mapping from $X$ to a universe $Y$, $y=f\left(x_{1}, x_{2}, \ldots, x_{r}\right)$. Then the extension principle allows us to define a fuzzy set $\tilde{B}$ in $Y$ by:

$$
\begin{aligned}
& \tilde{B}=\left\{\left(y, \mu_{\tilde{B}}(y)\right) \mid y=f\left(x_{1}, x_{2}, \ldots, x_{r}\right)\right. \\
& \left.\left(x_{1}, x_{2}, \ldots, x_{r}\right) \in X\right\}
\end{aligned}
$$

where

$$
\mu_{\tilde{B}}(y)=\left\{\sup \min _{\left(x_{1}, x_{2}, \ldots, x_{r}\right) \in f^{-1}(y)}\left\{\mu_{\tilde{A}_{1}}\left(x_{1}\right), \mu_{\tilde{A}_{2}}\left(x_{2}\right), \ldots, \mu_{\tilde{A}_{r}}\left(x_{r}\right)\right\} \text { if } f^{-1}(y) \neq \emptyset\right\}
$$

and $f^{-1}$ is the inverse of $f$.

For $=1$, the extension principle, of course, reduces to

$$
\tilde{B}=f(\tilde{A})=\left\{\left(y, \mu_{\tilde{B}}(y)\right) \mid y=f(x), x \in X\right\}
$$

where

$$
\begin{aligned}
& \mu_{\tilde{B}}(y)= \begin{cases}\min _{x \in f^{-1}(y)} \mu_{\tilde{A}}(x) & , f^{-1}(y) \neq \varnothing \\
0 & , \text { otherwise }\end{cases} \\
& \text { 1. }[\tilde{A} \oplus \tilde{B}]^{\alpha}=[\tilde{A}]^{\alpha}+[\tilde{B}]^{\alpha}=\left[\underline{A}^{\alpha}+\underline{B}^{\alpha}, \bar{A}^{\alpha}+\bar{B}^{\alpha}\right] \\
& \text { 2. } k[\tilde{A}]^{\alpha}= \begin{cases}{\left[k \underline{A}^{\alpha}, k \bar{A}^{\alpha}\right]} & , k \geq 0 \\
{\left[k \bar{A}^{\alpha}, k \underline{A}^{\alpha}\right]} & , k<0\end{cases}
\end{aligned}
$$

\subsubsection{Algebraic operations with triangular fuzzy numbers}

Product and subtraction are shown by $\oplus, \odot$ and $\ominus$, respectively.

Theorem 2.1: (Zimmermann, 1996) Let $\tilde{A}, \tilde{B} \in$ $\mathcal{F}(\mathbb{R})$, then: 


$$
\begin{gathered}
\text { 3. }[\tilde{A} \ominus \tilde{B}]^{\alpha}=[\tilde{A}]^{\alpha} \oplus(-1) \odot[\tilde{B}]^{\alpha} \\
\text { 4. }[\tilde{A} \odot \tilde{B}]^{\alpha}=\left[\min \left\{\underline{A}^{\alpha} \underline{B}^{\alpha}, \underline{A}^{\alpha} \bar{B}^{\alpha}, \bar{A}^{\alpha} \underline{B}^{\alpha}, \bar{A}^{\alpha} \bar{B}^{\alpha}\right\}, \max \left\{\underline{A}^{\alpha} \underline{B}^{\alpha}, \underline{A}^{\alpha} \bar{B}^{\alpha}, \bar{A}^{\alpha} \underline{B}^{\alpha}, \bar{A}^{\alpha} \bar{B}^{\alpha}\right\}\right]
\end{gathered}
$$

Proof: See Ma et al. (1999).

Definition 2.2.2: $\forall \alpha \in[0,1]$, we have $[\tilde{f}]^{\alpha}=\left(\underline{f}^{\alpha}, \bar{f}^{\alpha}\right)$.

Definition 2.2.3: $\forall \alpha \in[0,1]$, we have

$$
\begin{aligned}
& {[\dot{\tilde{f}}(x)]^{\alpha}=} \\
& {\left[\min \left\{\left(\underline{f}^{\alpha}\right)^{\prime},\left(\bar{f}^{\alpha}\right)^{\prime}\right\}, \max \left\{\left(\underline{f}^{\alpha}\right)^{\prime},\left(\bar{f}^{\alpha}\right)^{\prime}\right\}\right]}
\end{aligned}
$$

Definition2.2.4: The metric structure is given by the Hausdroff distance

$$
\begin{aligned}
& D: \in \mathcal{F}(\mathbb{R}) \times \in \mathcal{F}(\mathbb{R}) \rightarrow \mathbb{R}^{+} \cup\{0\} \\
& D(u, v)=\sup _{\alpha \in[0,1]} \max \left\{\left|\underline{u}^{\alpha}-v^{\alpha}\right|,\left|\bar{u}^{\alpha}-\bar{v}^{\alpha}\right|\right.
\end{aligned}
$$

$\left(R_{F}, D\right)$ is a complete metric space and the following properties are well known:

$$
\begin{gathered}
\begin{array}{c}
D(u+w, v+w)=D(u, v), \forall u, v, w \in \mathcal{F}(\mathbb{R}) \\
D(k \odot u, k \odot v)=|k| D(u, v), \forall k \in R, u, v \\
\in \mathcal{F}(\mathbb{R})
\end{array} \\
D(u+v, w+e) \leq D(u, w)+D(v, e) \\
, \forall u, v, w, e \in \mathcal{F}(\mathbb{R})
\end{gathered}
$$

Definition 2.2.5: (Bede and Stefanini, 2013). Let $\tilde{A}, \tilde{B} \in \mathcal{F}(\mathbb{R})$, the generalized Hukahara difference (gH-difference) of two fuzzy numbers $\tilde{A}$ and $\tilde{B}$ is the fuzzy number $\tilde{C} \in \mathcal{F}(\mathbb{R})$, if it exists such that

where

$$
\tilde{A} \ominus_{g H} \tilde{B}=\tilde{C} \Leftrightarrow\left\{\begin{array}{l}
\tilde{A}=\tilde{B} \oplus \tilde{C} \\
\tilde{B}=\tilde{A} \ominus \tilde{C}
\end{array}\right.
$$

$$
\tilde{A} \ominus \tilde{C}=\tilde{A} \oplus((-1) \odot \tilde{C})
$$

In terms of $\alpha$-cuts we have $\left[\tilde{A} \ominus_{g H} \tilde{B}\right]^{\alpha}=$ $\left[\min \left\{\underline{A}^{\alpha}-\underline{B}^{\alpha}, \underline{A}^{\alpha}-\bar{B}^{\alpha}\right\}, \max \left\{\underline{A}^{\alpha}-\underline{B}^{\alpha}, \underline{A}^{\alpha}-\bar{B}^{\alpha}\right\}\right]$. Each of following conditions guarantees the existence of $\tilde{C}=\tilde{A} \ominus_{g H} \tilde{B} \in \mathcal{F}(\mathbb{R})$ (see 24 for more details)

Definition 2.2.6: (Bede and Stefanini, 2013). Let $\tilde{f}=T \subset \mathbb{R} \rightarrow \mathcal{F}(\mathbb{R})$ and $t_{0} \in T$ be a fixed number, then $\tilde{f}$ is called Gh-differentiable at $t_{0} \in T$ if:

$$
\lim _{h \rightarrow 0} \frac{\tilde{f}\left(t_{0}+h\right) \ominus_{g H} \tilde{f}\left(t_{0}\right)}{h}=\tilde{f}_{g H}^{\prime}\left(t_{0}\right) \in \mathcal{F}(\mathbb{R}) .
$$

Theorem 2.2: Let $\tilde{f}=T \subset \mathbb{R} \rightarrow \mathcal{F}(\mathbb{R})$ be a fuzzy function, where $[\tilde{f}(t)]^{\alpha}=\left[\underline{f}^{\alpha}(t)-\bar{f}^{\alpha}(t)\right]$. Suppose that the functions $f^{\alpha}(t)$ and $\bar{f}^{\alpha}(t)$ are real-valued functions, differentiable and uniformly for $\alpha \in[0,1]$. Then the function $\tilde{f}(t)$ is gH-differentiable at a fixed $t \in T$ if and only if one of the following two cases hold: (a) $f^{\prime \alpha}(t)$ is increasing, $\bar{f}^{\prime \alpha}(t)$ is decreasing as functions of $\alpha$, and $f^{\prime 1}(t) \leq \bar{f}^{\prime 1}(t)$, or

(b) $f^{\prime \alpha}(t)$ is decreasing, $\bar{f}^{\prime \alpha}(t)$ is increasing as functions of $\alpha$, and $\bar{f}^{\prime 1}(t) \leq f^{\prime 1}(t)$.

Also, $\forall \alpha \in[0,1]$ we have

$$
\begin{aligned}
& {\left[f^{\prime}{ }_{g H}(t)\right]^{\alpha}=} \\
& {\left[\min \left\{\underline{f}^{\prime \alpha}(t), \bar{f}^{\prime \alpha}(t)\right\}, \max \left\{\underline{f}^{\prime \alpha}(t), \bar{f}^{\prime \alpha}(t)\right\}\right]}
\end{aligned}
$$

Proof: See Bede and Stefanini (2013).

Definition 2.2.7: (Bede and Stefanini, 2013) Let $\tilde{f}: T \subset \mathbb{R} \rightarrow \mathcal{F}(\mathbb{R})$ and $t_{0} \in T$. If $\underline{f}^{\alpha}(t), \bar{f}^{\alpha}(t)$ are both differentiable at $t_{0}$, then

1. $\tilde{f}$ is called (i)-gH-differentiable at $t_{0}$ if $\left[\widetilde{f}_{g H}^{\prime}\left(t_{0}\right)\right]^{\alpha}=\left[\underline{f}^{\prime \alpha}\left(t_{0}\right), \bar{f}^{\prime \alpha}\left(t_{0}\right)\right], \forall \alpha \in[0,1]$,

2. $\tilde{f}$ is called (ii)-gH-differentiable at $t_{0}$ if $\left[\widetilde{f}_{g H}^{\prime}\left(t_{0}\right)\right]^{\alpha}=\left[\bar{f}^{\prime \alpha}\left(t_{0}\right), \underline{f}^{\prime \alpha}\left(t_{0}\right)\right], \forall \alpha \in[0,1]$.

\subsection{Fuzzy functional}

At first, we define fuzzy vector space. The fuzzy vector space is denoted by $\mathcal{F}(\mathbb{R})$ and has following properties:

If $\tilde{A}, \tilde{B} \in \mathcal{F}(\mathbb{R})$, then

1. $\tilde{A} \oplus \tilde{B}=\tilde{C} \quad$, where $\tilde{C} \in \mathcal{F}(\mathbb{R})$

2. $\tilde{A} \oplus \tilde{B}=\tilde{B} \oplus \tilde{A}$

3. $\tilde{A} \oplus \tilde{0}=\tilde{0} \oplus \tilde{A}=\tilde{A}$

4. $\tilde{A} \oplus(-\tilde{A})=\tilde{0}$

5. $r \tilde{A} \in \mathcal{F}(\mathbb{R}), r \in \mathbb{R}$

6. $r(\tilde{A} \oplus \tilde{B})=r \tilde{A} \oplus r \tilde{B}$.

Definition 2.3.1: Let $\mathcal{F}(\mathbb{R})$ is the fuzzy vector space and $\tilde{f}=(\underline{f}, \bar{f}) \in \mathcal{F}(\mathbb{R})$ is a fuzzy function. We denote fuzzy $L_{1}-$ norm by $\|\cdot\|_{1}$ and is defined as follows:

$$
\|\tilde{f}\|_{1}=\|(\underline{f}, \bar{f})\|_{1}=|\underline{f}|+|\bar{f}|
$$

\section{Fuzzy Initial value problem (FIVP)}

In this section, at first, we consider the initial value problem (IVP).

$$
\left\{\begin{array}{l}
\dot{x}=f(t, x(t)), t \in\left[t_{0}, T\right] \\
x\left(t_{0}\right)=x_{0}
\end{array}\right.
$$

where $f:\left[t_{0}, T\right] \times \mathbb{R} \rightarrow \mathbb{R}$ is a continuous function defined on $\left[t_{0}, T\right]$ with $T>0$ and $x_{0} \in \mathbb{R}$. Suppose that the initial condition in (3.1) is uncertain and modelled by a fuzzy number, then we have the following fuzzy initial value problem:

$$
\left\{\begin{array}{l}
\dot{\tilde{x}}=f(t, \tilde{x}(t)), t \in\left[t_{0}, T\right] \\
\tilde{x}\left(t_{0}\right)=\tilde{x}_{0}
\end{array}\right.
$$


where $f:\left[t_{0}, T\right] \times \mathcal{F}(\mathbb{R}) \rightarrow \mathcal{F}(\mathbb{R})$ is a fuzzy valued function on $\left[t_{0}, T\right]$ with $T>0$ and $\tilde{x}_{0}=(\underline{x}(0), \bar{x}(0)) \in \mathcal{F}(\mathbb{R})$. To interpret the connection between (3.1) and (3.2) we refer to Mizukoshi et al. (2007) and Hullermeier (1999).

As we explained in "Introduction" section, some researchers have worked on numerical solution of fuzzy initial value problem FIVP. In this study, we have used a different approach for obtaining numerical solution of this FIVP.

\section{New approach}

We consider the following fuzzy initial value problem:

$$
\left\{\begin{array}{l}
\dot{\tilde{x}}(t)=a \tilde{x}(t), t \in[0, T] \\
\tilde{x}(0)=\tilde{x}_{0}
\end{array}\right.
$$

where $\tilde{x}(t)=(\underline{x}, \bar{x})$ and $\underline{x}, \bar{x}$ are left and right spread, respectively.

At first, we define the general error functional as follows:

Definition 4.1: We denote the general error functional by $E(\dot{\tilde{x}}(t), \tilde{x}(t), t)$ and define it as follows:

$$
E(\dot{\tilde{x}}(t), \tilde{x}(t), t)=\int_{0}^{T}\|\dot{\tilde{x}}(t) \ominus a \tilde{x}(t)\|_{1} d t
$$

We consider following optimization problem to solve fuzzy initial value problem (4.1).

$$
\begin{gathered}
\operatorname{Min} \int_{0}^{T}\|\dot{\tilde{x}}(t) \ominus a \tilde{x}(t)\|_{1} d t \\
\text { s.t } \\
\tilde{x}(0)=(\underline{x}(0), \bar{x}(0))
\end{gathered}
$$

Now, we explain that the optimal solution of optimization problem (4.3) is approximated solution of fuzzy initial value problem (4.1).

Definition 4.2: We define the $\alpha$-cuts as follows:

$$
\begin{aligned}
& {[\tilde{x}(t)]^{\alpha}=\left(\underline{x}^{\alpha}(t), \bar{x}^{\alpha}(t)\right)} \\
& {[\dot{\tilde{x}}(t)]^{\alpha}=\left(\underline{\dot{x}}^{\alpha}(t), \overline{\dot{x}}^{\alpha}(t)\right)}
\end{aligned}
$$

Lemma 4.1: If the function $h(x)$ is continuous on $[a, b]$ and $\int_{a}^{b}|h(x)| d x=0$, then $h(x)=0$.

Proof: We suppose that there exists any point $s \in(a, b)$ that $h(s) \neq 0$ and so, $|h(s)|>0$. Also, because $h(x)$ is continuous, so, $|h(x)|$ is continuous.

Then, there exist $0<r$ such that $\forall x \in$ $(s-r, s+r):|h(x)|>0$. So, we have:

$$
\begin{aligned}
\int_{a}^{b}|h(x)| d x=\int_{a}^{s-r}|h(x)| d x+\int_{s-r}^{s+r}|h(x)| d x \\
+\int_{s+r}^{b}|h(x)| d x>\int_{s-r}^{s+r}|h(x)| d x>0
\end{aligned}
$$

It is contradiction. So, $h(x)=0$.

Theorem 4.1: The necessary and sufficient condition for fuzzy initial value problem (4.1) to have solution $\tilde{x}(t)$ is:

$$
E(\dot{\tilde{x}}(t), \tilde{x}(t), t)=0
$$

Proof: It is sufficient to consider function $h(t)$ in lemma 4.1 as follows:

$$
h(t)=\|\dot{\tilde{x}}(t) \ominus a \tilde{x}(t)\|_{1}
$$

As the norm function $\|\cdot\|_{1}, \tilde{x}(\cdot)$ and $\dot{\tilde{x}}(\cdot)$ are continuous functions, so, according lemma 6.1 we have:

$$
\begin{aligned}
E(\dot{\tilde{x}}(t), \tilde{x}(t), t)= & \int_{0}^{T}\|\dot{\tilde{x}}(t) \ominus a \tilde{x}(t)\|_{1} d t=0 \\
& \Leftrightarrow\|\dot{\tilde{x}}(t) \ominus a \tilde{x}(t)\|_{1}=0
\end{aligned}
$$

Theorem 4.2: The necessary and sufficient condition for fuzzy initial value problem (4.1) to have solution $\tilde{x}^{*}(t)$ is that the optimization problem (4.3) has zero optimal amount.

Proof: According lemma 4.1 and theorem 6.1 that is clear.

So, according theorem 4.2, we can obtain the solution of fuzzy initial value problem (4.1) using solving the optimization problem (4.3). If the optimization problem (4.3) does not have zero solution then the best approximated solution is achieved for fuzzy initial value problem (4.1).

Now, we consider optimization problem (4.3)

$$
\begin{aligned}
& \text { Min } \int_{0}^{T}\|\dot{\tilde{x}}(t) \ominus a \tilde{x}(t)\|_{1} d t \\
& \text { s.t } \\
& \tilde{x}(0)=(\underline{x}(0), \bar{x}(0)) \\
& \text { As definition 4.2, we have: } \\
& {[\dot{x}(t)]^{\alpha}-a[\tilde{x}(t)]^{\alpha}=\left(\underline{\dot{x}}^{\alpha}(t)-a \bar{x}^{\alpha}(t), \overline{\dot{x}}^{\alpha}(t)-\right.} \\
& \left.a \underline{x}^{\alpha}(t)\right)=\left(\frac{\underline{x}^{\alpha}(t+\Delta)-\underline{x}^{\alpha}(t)}{\Delta}-a \bar{x}^{\alpha}(t), \frac{\bar{x}^{\alpha}(t+\Delta)-\bar{x}^{\alpha}(t)}{\Delta}-\right. \\
& \left.a \underline{x}^{\alpha}(t)\right)
\end{aligned}
$$

So, the optimization problem (4.3) is as follows:

$$
\begin{aligned}
& \operatorname{Min} \int_{0}^{T} \| \frac{\underline{x^{\alpha}(t+\Delta)-\underline{x}^{\alpha}(t)}}{\Delta}-a \bar{x}^{\alpha}(t), \frac{\bar{x}^{\alpha}(t+\Delta)-\bar{x}^{\alpha}(t)}{\Delta}- \\
& a \underline{x}^{\alpha}(t) \|_{1} d t \\
& \text { s.t } \\
& \tilde{x}(0)=\left(\underline{x_{0}}, \overline{x_{0}}\right)
\end{aligned}
$$

Now, we partition interval $[0, T]$ to $\mathrm{n}$ equal subintervals and define:

$t_{i}=\frac{i}{n} T \quad: i=0,1, \ldots . n$

So, we have: 


$$
\begin{aligned}
& \operatorname{Min} \int_{0}^{T} \| \frac{\underline{x}^{\alpha}(t+\Delta)-\underline{x}^{\alpha}(t)}{\Delta}-a \bar{x}^{\alpha}(t), \frac{\bar{x}^{\alpha}(t+\Delta)-\bar{x}^{\alpha}(t)}{\Delta}- \\
& a \underline{x}^{\alpha}(t) \|_{1} d t \\
& \text { s.t } \\
& \tilde{x}(0)=\left(\underline{x_{0}}, \overline{x_{0}}\right)
\end{aligned}
$$

Now, we let $\underline{x}\left(t_{i}\right)=\underline{x}_{i}, \underline{x}\left(t_{i}+\Delta\right)=\underline{x}_{i+1}$, $\bar{x}\left(t_{i}\right)=\bar{x}_{i}, \bar{x}\left(t_{i}+\Delta\right)=\bar{x}_{i+1}, \Delta=\frac{T}{n}$. So, if $n$ is increasing to a great number, then the integral in the optimization problem is approximated by a sigma. Now, according Riemann integral definition we can approximate the optimization problem by below problem:

$$
\begin{aligned}
& \operatorname{Min} \frac{T}{n} \sum_{i=1}^{n} \|\left(\frac{n}{T}\left(\underline{x}_{i+1}^{\alpha}-\underline{x}_{i}^{\alpha}\right)-a \bar{x}_{i}^{\alpha}, \frac{n}{T}\left(\bar{x}_{i+1}^{\alpha}-\right.\right. \\
& \left.\left.\bar{x}_{i}^{\alpha}\right)-a \underline{x}_{i}^{\alpha}\right) \|_{1} \\
& \text { s.t } \\
& \tilde{x}(0)=\left(x_{0}, \overline{x_{0}}\right)
\end{aligned}
$$

And using the $L_{1-}$ norm definition we have:

$\operatorname{Min} \frac{T}{n} \sum_{i=1}^{n}\left|\frac{n}{T}\left(\underline{x}_{i+1}^{\alpha}-\underline{x}_{i}^{\alpha}\right)-a \bar{x}_{i}^{\alpha}\right|+\mid \frac{n}{T}\left(\bar{x}_{i+1}^{\alpha}-\right.$

$\left.\bar{x}_{i}^{\alpha}\right)-a \underline{x}_{i}^{\alpha}$

s.t

$\tilde{x}(0)=\left(\underline{x_{0}}, \overline{x_{0}}\right)$

by relations $\quad\left|\frac{n}{T}\left(f_{i+1}-f_{i}\right)-a f_{i}\right|=r_{i}+s_{i}$ and $\left|\frac{n}{T}\left(\alpha_{i+1}-\alpha_{i}\right)+a \beta_{i}\right|=v_{i}+w_{i}$ we have:

$$
\begin{aligned}
& \operatorname{Min} \frac{T}{n} \sum_{i=1}^{n}\left(\left|r_{i}-s_{i}\right|+\left|v_{i}-w_{i}\right|\right) \\
& \text { s.t } \\
& r_{i}-s_{i}=\frac{n}{T}\left(\underline{x}_{i+1}^{\alpha}-\underline{x}_{i}^{\alpha}\right)-a \bar{x}_{i}^{\alpha} \\
& v_{i}-w_{i}=\frac{n}{T}\left(\bar{x}_{i+1}^{\alpha}-\bar{x}_{i}^{\alpha}\right)-a \underline{x_{i}^{\alpha}} \\
& \tilde{x}(0)=\left(\underline{x_{0}}, \overline{x_{0}}\right) \\
& r_{i}, s_{i}, v_{i}, w_{i} \geq 0 ; i=1,2, \ldots, n
\end{aligned}
$$

But, this problem is a nonlinear programming problem. Finally, to change this problem to a linear problem we use relations $\left|r_{i}-s_{i}\right|=r_{i}+s_{i}$ and $\left|v_{i}-w_{i}\right|=v_{i}+w_{i}$. So, we have this linear programming problem:

$$
\begin{aligned}
& \operatorname{Min} \frac{T}{n} \sum_{i=1}^{n}\left(r_{i}+s_{i}\right)+\left(v_{i}+w_{i}\right) \\
& \text { s.t } \\
& r_{i}-s_{i}=\frac{n}{T}\left(\underline{x}_{i+1}^{\alpha}-\underline{x}_{i}^{\alpha}\right)-a \bar{x}_{i}^{\alpha} \\
& v_{i}-w_{i}=\frac{n}{T}\left(\bar{x}_{i+1}^{\alpha}-\bar{x}_{i}^{\alpha}\right)-a \underline{x}_{i}^{\alpha} \\
& \tilde{x}(0)=\left(\underline{x_{0}}, \overline{x_{0}}\right) \\
& r_{i}, s_{i}, v_{i}, w_{i} \geq 0 ; i=1,2, \ldots, n \\
& r_{i}, s_{i}, v_{i}, w_{i} \geq 0 ; i=1,2, \ldots, n
\end{aligned}
$$

As we explained in section 6, the optimal solution of this linear programming problem is the approximated solution of fuzzy initial value problem
(FIVP). The accurate of solution is getting to improve as $n$ is increasing.

\section{Numerical experiments}

In this section, we present some experiments to show performance and accuracy of presented method.

Example 5.1: Consider the following fuzzy initial value problem:

$$
\left\{\begin{array}{l}
y^{\prime}(t)=-y(t) \\
y(0)=(0.960 .04 \alpha, 1.01-0.01 \alpha)
\end{array}\right.
$$

the exact solution at $t=1$ is

$$
\begin{aligned}
& Y(1, \alpha)=\left((0.96+0.04 \alpha) e^{-1},(1.01-\right. \\
& \left.0.01 \alpha) e^{-1}\right), \quad 0 \leq \alpha \leq 1
\end{aligned}
$$

This problem has been solved in (Allahviranloo, 2004). We have solved this problem using new approach for $n=10$. The approximated solution at $t=1$ is shown in table 1 . Also, $\mathrm{Y}, \overline{\mathrm{Y}}$ are exact left and right spreads and $y, \bar{y}$ are approximate left and right spreads, respectively.

Example 5.2: Consider the following fuzzy initial value problem:

$$
\left\{\begin{array}{l}
y^{\prime}(t)=y(t)(1-2 t) \\
y(0)=y_{0}
\end{array}\right.
$$

where

$$
y_{0}(s)=\left\{\begin{array}{cr}
0, & s<-1 / 2 \\
1-4 s^{2}, & -1 / 2 \leq s \leq 1 / 2 \\
0, & 1 / 2<s
\end{array}\right.
$$

The analytical solution for this problem is:

$$
[y(t)]^{\alpha}=\left\{\left(-\frac{\sqrt{1-\alpha}}{2}\right) e^{\left.t-t^{2}\right)},\left(\frac{\sqrt{1-\alpha}}{2}\right) e^{t-t^{2}}\right\}
$$

We have solved this problem using new approach for $n=10$. The numerical solution is high accurate and the accuracy of the numerical solution is increasing as $\mathrm{n}$ is increasing (Table 2). We have shown the exact and approximated solutions at $t=1$ (Figs. 2-4).

\section{Conclusion}

In this paper, we have presented a new approach is based on an optimization problem. In fact, we transform fuzzy initial value problem to a linear programming problem. The optimal solution of this linear programming problem is approximated solution of fuzzy initial value problem. This new approach is high accurate and very simple. Since, it does not need any differentiability of the fuzzy functions, so we can use it to solve fuzzy nonsmooth systems. 
Table 1: The comparison between exact and approximate solution at $t=1, n=10$ in example 5.1

\begin{tabular}{|c|c|c|c|c|c|c|}
\hline$\alpha$ & $\underline{y}$ & $\underline{Y}$ & \multicolumn{1}{c}{ Error } & $\bar{y}$ & \multicolumn{1}{c}{$\bar{Y}$} & Error \\
\hline 0 & 0.3365 & 0.3532 & 0.0167 & 0.3541 & 0.3716 & 0.0175 \\
\hline 0.1 & 0.3379 & 0.3546 & 0.0167 & 0.3537 & 0.3712 & 0.0175 \\
\hline 0.2 & 0.3393 & 0.3561 & 0.0168 & 0.3534 & 0.3708 & 0.0174 \\
\hline 0.3 & 0.3407 & 0.3576 & 0.0169 & 0.3530 & 0.3705 & 0.0175 \\
\hline 0.4 & 0.3421 & 0.3591 & 0.0170 & 0.3527 & 0.3701 & 0.0174 \\
\hline 0.5 & 0.3435 & 0.3605 & 0.0170 & 0.3523 & 0.3697 & 0.0174 \\
\hline 0.6 & 0.3449 & 0.3620 & 0.0171 & 0.3520 & 0.3694 & 0.0174 \\
\hline 0.7 & 0.3463 & 0.3635 & 0.0172 & 0.3516 & 0.3690 & 0.0174 \\
\hline 0.8 & 0.3477 & 0.3649 & 0.0172 & 0.3513 & 0.3686 & 0.0173 \\
\hline 0.9 & 0.3492 & 0.3664 & 0.0172 & 0.3509 & 0.3682 & 0.0173 \\
\hline 1 & 0.3506 & 0.3679 & 0.0173 & 0.3506 & 0.3679 & 0.0173 \\
\hline
\end{tabular}

Table 2: The comparison between exact and approximate solution at $t=1, n=10$ in example 5.2

\begin{tabular}{|c|c|c|c|c|c|c|}
\hline$\alpha$ & $\underline{y}$ & $\underline{Y}$ & Error & $\bar{y}$ & $\bar{Y}$ & Error \\
\hline 0 & -0.5907 & -0.5000 & 0.0907 & 0.5907 & 0.5000 & 0.0907 \\
\hline 0.1 & -0.5604 & -0.4743 & 0.0861 & 0.5604 & 0.4743 & 0.0861 \\
\hline 0.2 & -0.5283 & -0.4472 & 0.0811 & 0.5283 & 0.4472 & 0.0811 \\
\hline 0.3 & -0.4942 & -0.4183 & 0.0759 & 0.4942 & 0.4183 & 0.0759 \\
\hline 0.4 & 0.4575 & -0.3873 & 0.0702 & 0.4575 & 0.3873 & 0.0702 \\
\hline 0.5 & -0.4177 & -0.3536 & 0.0641 & 0.4177 & 0.3536 & 0.0641 \\
\hline 0.6 & -0.3736 & -0.3162 & 0.0574 & 0.3736 & 0.3162 & 0.0574 \\
\hline 0.7 & -0.3235 & -0.2739 & 0.0496 & 0.3235 & 0.2739 & 0.0496 \\
\hline 0.8 & -0.2642 & -0.2236 & 0.0406 & 0.2642 & 0.2236 & 0.0406 \\
\hline 0.9 & -0.1868 & -.1581 & 0.0287 & 0.1868 & .1581 & 0.0287 \\
\hline 1 & 0 & 0 & 0 & 0 & 0 & 0 \\
\hline
\end{tabular}

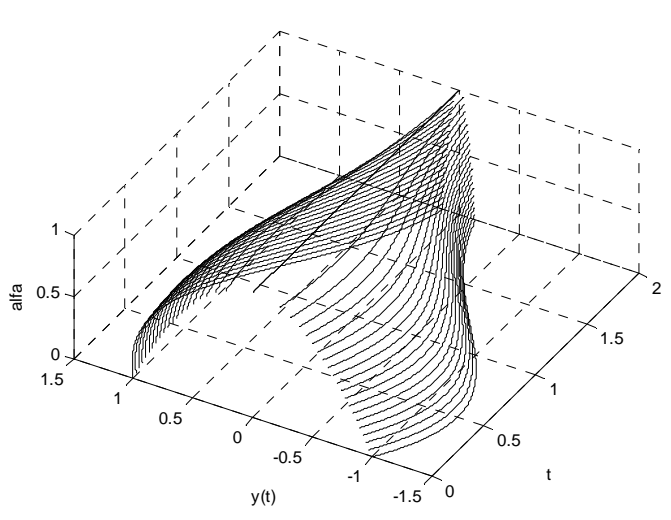

Fig. 2: The exact solution of example 5.2



Fig. 3: The approximate solution of example 5.2

$$
(\alpha=0, .1, .2, \ldots, 1)
$$



Fig. 4: The approximate solution of example 5.2

\section{References}

Abbasbandy S and Viranloo TA (2002). Numerical solutions of fuzzy differential equations by Taylor method. Computational Methods in Applied Mathematics, 2(2): 113-124.

Abbasbandy S, Viranloo TA, Lopez-Pouso 0 and Nieto JJ (2004). Numerical methods for fuzzy differential inclusions. Computers and Mathematics with Applications, 48(10): 16331641.

Allahviranloo T (2004). Numerical methods for fuzzy system of linear equations. Applied Mathematics and Computation, 155(2): 493-502.

Allahviranloo T (2005a). Successive over relaxation iterative method for fuzzy system of linear equations. Applied Mathematics and Computation, 162(1): 189-196. 
Allahviranloo T (2005b). The Adomian decomposition method for fuzzy system of linear equations. Applied Mathematics and Computation, 163(2), 553-563.

Allahviranloo T, Ahmady N and Ahmady E (2006). Two step method for fuzzy differential equations. In International Mathematical Forum, 1(17-20): 823-832.

Allahviranloo T, Ahmady $\mathrm{N}$ and Ahmady E (2007). Numerical solution of fuzzy differential equations by predictor-corrector method. Information Sciences, 177(7): 1633-1647.

Bede B and Gal SG (2005). Generalizations of the differentiability of fuzzy-number-valued functions with applications to fuzzy differential equations. Fuzzy Sets and Systems, 151(3): 581599.

Bede B and Stefanini L (2013). Generalized differentiability of fuzzy-valued functions. Fuzzy Sets and Systems, 230: 119-141.

Diamond P (2000). Stability and periodicity in fuzzy differential equations. IEEE Transactions on Fuzzy Systems, 8(5): 583-590.

Dubios D and Prade H (1982). Towards Fuzzy Differential Calclus: Part 3, differentiation. Fuzzy Sets and Systems (8): 225-233.

Friedman M, Ming M and Kandel A (1998). Fuzzy linear systems. Fuzzy Sets and Systems, 96(2): 201-209.

Hüllermeier E (1999). Numerical methods for fuzzy initial value problems. International Journal of
Uncertainty, Fuzziness and Knowledge-Based Systems, 7(05): 439-461.

Kaleva $O$ (1987). Fuzzy differential equations. Fuzzy Sets and Systems, 24(3): 301-317.

Kaleva 0 (1990). The Cauchy problem for fuzzy differential equations. Fuzzy sets and systems, 35(3): 389-396.

Ma M, Friedman M and Kandel A (1999). Numerical solutions of fuzzy differential equations. Fuzzy Sets and Systems, 105(1): 133-138.

Mizukoshi MT, Barros LC, Chalco-Cano Y, RománFlores $H$ and Bassanezi RC (2007). Fuzzy differential equations and the extension principle. Information Sciences, 177(17): 3627-3635.

Puri ML and Ralescu DA (1983). Differentials of fuzzy functions. Journal of Mathematical Analysis and Applications, 91(2): 552-558.

Seikkala S (1987). On the fuzzy initial value problem. Fuzzy Sets and Systems, 24(3): 319-330.

Vroman A, Deschrijver G and Kerre EE (2008). Using parametric functions to solve systems of linear fuzzy equations with a symmetric matrix. International Journal of Computational Intelligence Systems, 1(3): 248-261.

Zadeh LA (1965). Fuzzy sets. Information and Control, 8(3): 338-353.

Zimmermann HJ (1996). Fuzzy Control. In Fuzzy Set Theory-and Its Applications. Springer Netherlands: 203-240. 